\title{
CALORIMETRIC STUDY OF THE DIGITAL BLOOD FLOW IN RHEUMATOID ARTHRITIS*
}

\author{
BY \\ MORSI MOHAMED ARAB $\dagger$, GEORGE HOLDEN, AND EDNA DE RAMOS \\ From the Departments of Experimental Medicine and Physical Medicine, Guy's Hospital, London
}

\begin{abstract}
Vascular changes in rheumatoid arthritis have been receiving increasing attention in the last few years. It is well known that cold hands are common in patients with rheumatoid arthritis and a peripheral circulatory disturbance has been considered to be one of the aetiological factors of the disease since the report of the Empire Rheumatism Council (1950), which showed that 40 per cent. of the 532 patients studied had a history of cold hands before other symptoms of the disease started, as opposed to 26 per cent. of the controls. It has been argued that the joints most affected are those of the hands and feet, so that it is probable that chilling of these joints in the presence of an inadequate circulatory response may induce greater susceptibility to damage by some agents already present in the tissues of the susceptible patients (Copeman, 1955).
\end{abstract}

From the pathological studies so far carried out, three types of vascular changes are described:

(1) A subacute vasculitis of the small vessels of the muscles reported by Sokoloff, Wilens, and Bunim (1951), and of the heart, muscles, and nerve sheaths by Cruickshank (1954).

(2) A severe widespread necrotizing arteritis of the large vessels indistinguishable from that seen in polyarteritis nodosa, observed by Aronoff, Johnson, and Dworkin (1960), Graef, Hickey, and Altmann (1949), Levin, Rivo, Scott, Figueroa, Fred, and Barrett (1953), and Schmid, Cooper, Ziff, and McEwen (1961).

(3) Obliterative endarteritis of the digital arteries, reported by Bywaters (1957). This was associated with visceral involvement, and it appeared to be morphologically different from polyarteritis nodosa.

Furthermore, post mortem studies carried out by Virtama (1959) sometimes showed obliteration and post-stenotic dilatation of the digital arteries with arterial dilatation near the bone erosions. Scott, Hourihane, Doyle, Steiner, Laws, Dixon, and

\footnotetext{
* Paper read to the Heberden Society on December 6, 1963.

+ Present address: Department of Medicine, University of Alexandria, Egypt.
}

Bywaters (1961), using brachial arteriography in selected rheumatoid patients showing manifestations suggestive of arterial disease, reported occlusion or distortion in digital arteries and confirmed the presence of intimal thickening by subsequent histological examination.

A vasomotor defect in cases of rheumatoid arthritis was described by Woodmansey (1951), who reported an intermediate position of these patients' responses to indirect or reflex heating between the normal controls and definite cases of occlusive vascular disease. These cases were tested by heating one foot and recording skin temperatures at other sites. Later, Beattie and Woodmansey (1953) described a better vasodilator response to indirect heating after the intravenous infusion of ACTH. Again, Catchpole, Jepson, and Kellgren (1954) studied selected patients suffering from rheumatoid arthritis and showing some evidence of cold cyanosed fingers and Raynaud's phenomena or acrocyanosis, and reported somewhat reduced peripheral vascular response to body heating in five out of seven cases. A better response was reported after cortisone therapy.

The object of the present work was to study digital blood flow in rheumatoid arthritis using a continuous flow calorimetry technique, which had been made available by a calorimeter introduced by Butterfield (1964), and subsequently used for the study of different abnormalities of the digital blood flow (Arab, 1964). The design of the experiment included:

(1) Random selection of the rheumatoid cases as regards the presence of peripheral circulatory symptoms.

(2) Quantitative estimation of digital blood flow in $\mathrm{ml}$./min. per unit of finger volume, rather than heat elimination: $(a)$ at rest, $(b)$ during temporary arterial obstruction, $(c)$ during reactive hyperaemia, $(d)$ after indirect body heating.

(3) Study of digital blood flow with a cold or warm local environment. 
(4) Comparison with normal controls and patients with peripheral vascular disease.

\section{Material}

Eighteen rheumatoid arthritic patients were included. There were eleven females and seven males, with ages ranging from 31 to 62 years, the duration of the disease being between 15 months and 16 years. Some were in-patients and others were out-patients.

The twelve control subjects comprised six males and six females; their ages ranged from 30 to 56 years and all were healthy or suffered from minor conditions not affecting the vascular system.

\section{Apparatus and Technique}

Digital blood flows were recorded simultaneously for two middle fingers of each subject by the continuous water flow calorimeter (Butterfield) under two different controlled water temperatures. Twelve rheumatoid cases and eight controls were tested at a temperature of $20^{\circ} \mathrm{C}$., and six rheumatoid cases and four controls were tested at $28^{\circ} \mathrm{C}$. The water flow through the calorimeter was kept steady at $280 \mathrm{ml} . / \mathrm{min}$. in all experiments by means of flowmeters. The temperature of the water was maintained to within $0.5^{\circ} \mathrm{C}$. in each test. The blood flows were recorded by a multiple channel recording galvanometer.

With the subject sitting comfortably, tests were done in four stages:

(a) The patient remained at rest for 20 minutes in order to follow the waves of vasodilatation and vasoconstriction as changes in blood flow. (The first 5 minutes were discounted to allow thermal equilibrium to take place.)

(b) Arterial ligature was carried out with a rubber-band tourniquet applied lightly around the finger before it was introduced into the calorimeter and held by a plastic ring. Tight ligation was applied when required for 8 minutes.

(c) Standardized reactive hyperaemia was brought about by sudden release of the ligature applied for the previous stage. The peak blood flow was achieved in 1 to 3 minutes and was recorded. The blood flow was then followed for 15 to 20 minutes more.

(d) Sympathetic release by indirect body heating was carried out with the patient in a warm bath at $43-44^{\circ} \mathrm{C}$., the two lower limbs and lower trunk being in the water and the rest of the body covered.

Calibration was done at each experiment by means of an electric wire heat generator.
Calculations of the blood flows were carried out according to the principle described by Butterfield with slight changes necessitated by the few modifications introduced into the technique itself (Arab, 1964). Thus the blood flow could be calculated by the equation

$$
F=\left(\frac{d / e}{t-c}\right) v,
$$

where $F$ is the blood flow in ml. per minute per cc. finger volume

$d$ deflection recorded for the finger

$e$ is the deflection equivalent to one calorie per minute, produced by the heat generator

$t$ is the oral temperature representing arterial blood temperature

$c$ is the temperature of the calorimeter (taken as equal to venous temperature)

$v$ is the volume of the finger in cc.

The clinical grading of the cases was carried out according to both the anatomical stage (Steinbrocker, Traeger, and Batterman, 1949) and the functional stage (M.R.C., 1955) of the disease. Specific laboratory tests were carried out, including the Waaler-Rose differential sheep cell agglutination test as modified by Greenbury (1957). The latexfixation test by slide technique was also carried out in most cases.

\section{Results}

Table I (opposite) shows the results of the laboratory studies on the eighteen rheumatoid patients, together with other relevant data.

\section{Digital Blood Flow at an Environmental Temperature of $20^{\circ}$ C. (Fig. 1, opposite)}

Table II (overleaf) shows the results of the digital blood flow (DBF) calculated as $\mathrm{ml} . / \mathrm{min}$./cc. finger volume in the twelve rheumatoid cases tested at this temperature. For each case, two figures are included under each of the four phases of the test, one representing the right hand digit and the other the left hand digit. Thus, at this temperature, the maximal flow after indirect heating (MBF) ranged between 0.232 and 0.633 (average of $0.403 \pm 0.023^{*}$ ) $\mathrm{ml}$. blood per min. per cc. finger volume.

At the stage of standard reactive hyperaemia (SRH) the DBF ranged between 0.030 and 0.267 $\mathrm{ml}$./min./cc. (average 0.107 $\pm 0 \cdot 014^{*}$ ).

At the resting phase the DBF ranged from 0.011 to 0.391 (average $0.117 \pm 0.023^{*}$ ) for the values of vasodilatation (VD) at $20^{\circ} \mathrm{C}$., and between 0.011 and 0.135 (average $0.045 \pm 0.003^{*}$ ) for the values of vasoconstriction (VC) at the same temperature.

\footnotetext{
* Mean \pm Standard Error.
} 
TABLE I

CLINICAL AND LABORATORY DATA FOR EIGHTEEN CASES OF RHEUMATOID ARTHRITIS

\begin{tabular}{|c|c|c|c|c|c|c|c|c|c|}
\hline $\begin{array}{l}\text { Case } \\
\text { No. }\end{array}$ & $\begin{array}{c}\text { Age } \\
\text { (yrs) }\end{array}$ & Sex & $\begin{array}{l}\text { Duration } \\
\text { of Disease } \\
\text { (yrs) }\end{array}$ & $\begin{array}{l}\text { Stage of } \\
\text { Disease }\end{array}$ & $\begin{array}{c}\text { Functional } \\
\text { Grade }\end{array}$ & $\begin{array}{c}\text { Hb } \\
\text { per cent. }\end{array}$ & $\begin{array}{c}\text { Erythrocyte } \\
\text { Sedimentation } \\
\text { Rate }\end{array}$ & $\begin{array}{c}\text { Latex- } \\
\text { Fixation } \\
\text { Test }\end{array}$ & $\begin{array}{c}\text { Differential } \\
\text { Agglutination } \\
\text { Titre }\end{array}$ \\
\hline $\begin{array}{r}1 \\
2 \\
3 \\
4 \\
5 \\
6 \\
7 \\
8 \\
9 \\
10 \\
11 \\
12 \\
13 \\
14 \\
15 \\
16 \\
17 \\
18\end{array}$ & $\begin{array}{l}55 \\
51 \\
49 \\
45 \\
43 \\
62 \\
43 \\
40 \\
39 \\
31 \\
46 \\
57 \\
40 \\
39 \\
54 \\
43 \\
47 \\
62\end{array}$ & $\begin{array}{l}\mathbf{F} \\
\mathbf{F} \\
\mathbf{F} \\
\mathbf{F} \\
\mathbf{F} \\
\mathbf{F} \\
\mathbf{F} \\
\mathbf{M} \\
\mathbf{M} \\
\mathbf{M} \\
\mathbf{M} \\
\mathbf{M} \\
\mathbf{M} \\
\mathbf{M} \\
\mathbf{F} \\
\mathbf{F} \\
\mathbf{F}\end{array}$ & $\begin{array}{c}16 \\
4 \\
4 \\
17 \text { mths } \\
8 \\
11 \\
3 \\
9 \\
8 \\
4 \\
17 \text { mths } \\
2 \\
15 \text { mths } \\
8 \\
4 \\
5 \\
8 \\
10\end{array}$ & $\begin{array}{l}\text { II } \\
\text { II } \\
\text { II } \\
\text { II } \\
\text { III } \\
\text { III } \\
\text { I } \\
\text { III } \\
\text { II } \\
\text { III } \\
\text { III } \\
\text { III } \\
\text { II } \\
\text { II } \\
\text { II } \\
\text { II } \\
\text { II } \\
\text { III }\end{array}$ & $\begin{array}{l}2 \\
2 \\
2 \\
2 \\
3 \\
3 \\
1 \\
2 \\
2 \\
3 \\
3 \\
3 \\
2 \\
2 \\
2 \\
2 \\
2 \\
3\end{array}$ & $\begin{array}{r}78 \\
95 \\
90 \\
89 \\
94 \\
85 \\
90 \\
91 \\
100 \\
85 \\
84 \\
100 \\
91 \\
99 \\
110 \\
87 \\
80\end{array}$ & $\begin{array}{r}25 \\
25 \\
32 \\
9 \\
36 \\
10 \\
27 \\
18 \\
23 \\
6 \\
30 \\
52 \\
20 \\
23 \\
14 \\
8 \\
22 \\
20\end{array}$ & $\begin{array}{l}- \\
\bar{z} \\
+ \\
+ \\
+ \\
+ \\
+ \\
= \\
+ \\
+ \\
+ \\
+ \\
+ \\
+ \\
+\end{array}$ & $\begin{array}{l}1=64, \text { etc. } \\
00 \\
1: 8 \\
1: 128 \\
1: 8 \\
1: 512 \\
1: 8 \\
1: 64 \\
0 \\
1: 16 \\
1: 64 \\
1: 64 \\
1: 32 \\
1: 16 \\
= \\
-\end{array}$ \\
\hline
\end{tabular}

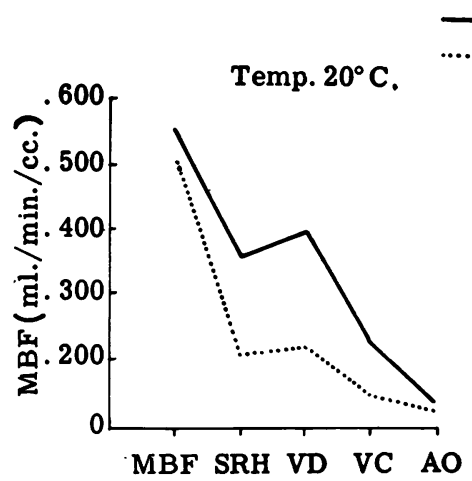

Normal

Rheumatoid
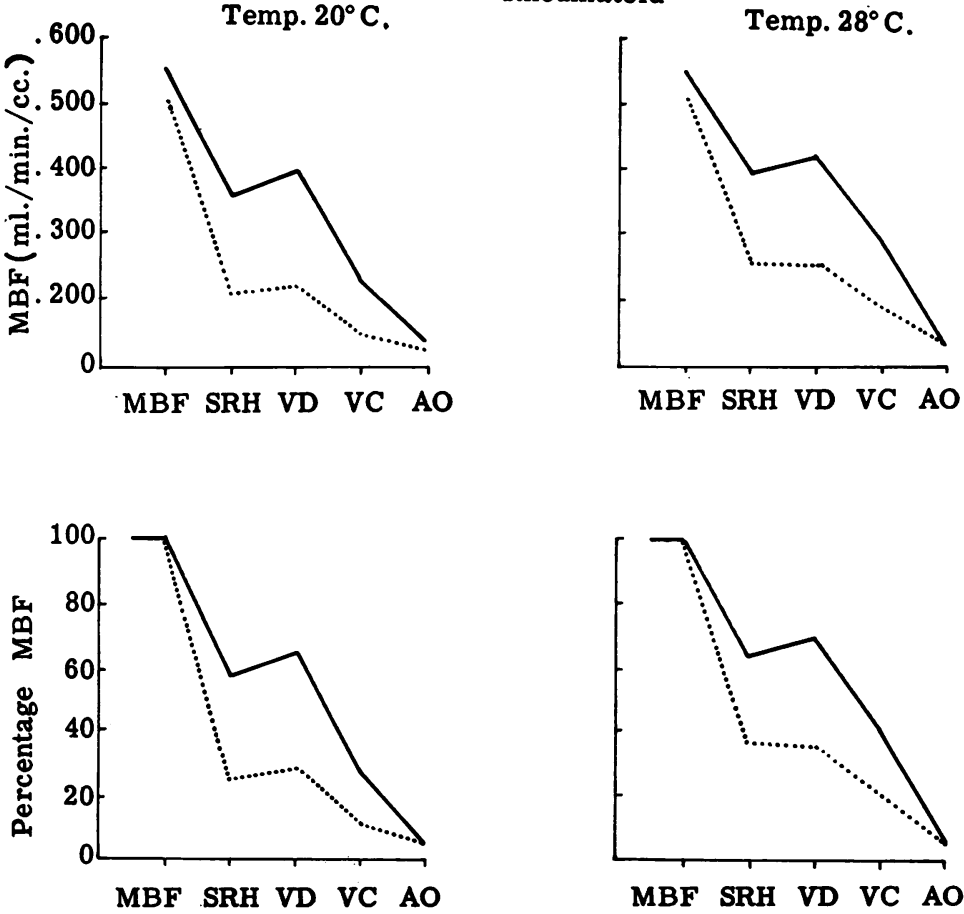

PHASE OF DIGTTAL BLOOD FLOW

Fig. 1.-Mean digital blood flow in normal subjects and rheumatoid arthritics at $20^{\circ}$ and $28^{\circ} \mathrm{C}$. Above: as $\mathrm{ml} . / \mathrm{min} . / \mathrm{cc}$. finger volume. Below: as percentage related to maximum blood flow (MBF). 
DigitAL BLOOD FLOW AT $20^{\circ}$ C. IN TWELVE CASES OF RHEUMATOID ARTHRITIS, BY SEX

\begin{tabular}{|c|c|c|c|c|c|c|c|c|}
\hline \multirow{2}{*}{$\operatorname{Sex}$} & \multirow{2}{*}{ Case No. } & \multirow{2}{*}{\multicolumn{2}{|c|}{ Age (yrs) }} & \multicolumn{5}{|c|}{ Digital Blood Flow (nl./min./cc.) } \\
\hline & & & & MBF & SRH & VD & VC & $\mathrm{AO}$ \\
\hline \multirow[t]{4}{*}{ Female } & $\begin{array}{l}1 \\
2 \\
3 \\
4 \\
5 \\
6 \\
7 \\
8\end{array}$ & $i$ & $\begin{array}{l}55 \\
51 \\
49 \\
45 \\
43 \\
62 \\
43 \\
40\end{array}$ & $\begin{array}{l}.410 \\
.430 \\
.0326 \\
.262 \\
.347 \\
.328 \\
.536 \\
.555 \\
.475 \\
.446 \\
.377 \\
.327 \\
.566 \\
.495 \\
.379 \\
.334\end{array}$ & $\begin{array}{l}.070 \\
.084 \\
.043 \\
.068 \\
.030 \\
.046 \\
.133 \\
.158 \\
.117 \\
.063 \\
.061 \\
.074 \\
.267 \\
.267 \\
.038 \\
.037\end{array}$ & $\begin{array}{l}.080 \\
.073 \\
.072 \\
.072 \\
.093 \\
.044 \\
.157 \\
.069 \\
.153 \\
.061 \\
.035 \\
.021 \\
.277 \\
.294 \\
.011 \\
.011\end{array}$ & $\begin{array}{l}.024 \\
.045 \\
.019 \\
.046 \\
.024 \\
.037 \\
.040 \\
.031 \\
.036 \\
.051 \\
.035 \\
.017 \\
.100 \\
.135 \\
.011 \\
.011\end{array}$ & $\begin{array}{l}.010 \\
.015 \\
.007 \\
.035 \\
.013 \\
.010 \\
.023 \\
.016 \\
.020 \\
.015 \\
.013 \\
.017 \\
.010 \\
.009 \\
.006 \\
.007\end{array}$ \\
\hline & Mean & - & $\ldots$ & .412 & .097 & .095 & .041 & .019 \\
\hline & S.D. & $\cdots$ & $\cdots$ & .092 & .075 & .080 & .032 & .007 \\
\hline & S.E. & . & $\cdots$ & .023 & .018 & .020 & .008 & .002 \\
\hline \multirow[t]{4}{*}{ Male } & $\begin{array}{r}9 \\
10 \\
11 \\
12\end{array}$ & & $\begin{array}{l}39 \\
31 \\
46 \\
57\end{array}$ & $\begin{array}{l}.633 \\
.558 \\
.233 \\
.288 \\
.423 \\
.436 \\
.282 \\
.232\end{array}$ & $\begin{array}{l}\cdot 132 \\
\cdot 188 \\
\cdot 103 \\
\cdot 167 \\
.055 \\
.068 \\
\cdot 177 \\
\cdot 133\end{array}$ & $\begin{array}{l}.391 \\
.366 \\
.102 \\
.206 \\
.033 \\
.029 \\
.088 \\
.076\end{array}$ & $\begin{array}{l}.092 \\
.075 \\
.054 \\
.063 \\
.018 \\
.019 \\
.045 \\
.052\end{array}$ & $\begin{array}{l}.029 \\
.034 \\
.035 \\
.036 \\
.011 \\
.009 \\
.032 \\
.039\end{array}$ \\
\hline & Mean & . & $\cdots$ & $\cdot 386$ & $\cdot 128$ & $\cdot 161$ & .052 & .028 \\
\hline & S.D. & . & . & $\cdot 152$ & .050 & $\cdot 144$ & .004 & .011 \\
\hline & S.E. & $\ldots$ & $\cdots$ & .054 & .019 & .051 & .002 & .004 \\
\hline \multirow{3}{*}{ Total } & Mean & . & . & .403 & $\cdot 107$ & $\cdot 117$ & .045 & .022 \\
\hline & S.D. & $\ldots$ & . & .113 & .070 & $\cdot 109$ & .017 & .011 \\
\hline & S.E. & . & $\ldots$ & .023 & .014 & .023 & .003 & .002 \\
\hline
\end{tabular}

Thus the average difference between waves of vasoconstriction (VC) and vasodilatation (VD) was $0.072 \mathrm{ml} . / \mathrm{min} . / \mathrm{cc}$.

At the stage of arterial obstruction (AO) the DBF ranged between 0.006 and 0.039 (average $\left.0.022 \pm 0.002^{*}\right)$. Thus the difference between the resting vasoconstriction and obstructed state was $0.023 \mathrm{ml} . / \mathrm{min} . / \mathrm{cc}$.

Table III (opposite) shows the corresponding figures for DBF in eight normal controls tested at the same temperature.

Table IV (opposite) summarizes the average values for DBF in cases of rheumatoid arthritis compared with normal controls at different phases of the test, as well as the percentages that represent the same values related to the $\mathrm{MBF}$ after indirect body heating; the latter being considered as the maximum 100 per cent. achievable flow in the digit.

\section{Digital Blood Flow at an Environmental Temperature of $28^{\circ}$ C. (Fig. 1)}

Table V (opposite) shows the DBF for the six rheumatoid patients tested at this temperature. The MBF ranged between 0.252 and $0.623 \mathrm{ml} . / \mathrm{min}$./cc. (average $0 \cdot 411 \pm 0 \cdot 039$ ). At SRH it ranged between $0 \cdot 106$ and $0 \cdot 262$ (average $0 \cdot 158 \pm 0 \cdot 015$ ). At the resting stage it ranged between 0.067 and 0.276 (average $0 \cdot 151 \pm 0 \cdot 020$ ) for the vasodilated condition, and between 0.052 and 0.212 (average $0.092 \pm 0.014$ ) for the vasoconstricted condition. Thus the difference representing the vasodilatationvasoconstriction wave at this temperature was $0.059 \mathrm{ml} . / \mathrm{min} . / \mathrm{cc}$. On arterial obstruction, the DBF ranged between 0.007 and $0.82 \mathrm{ml} . / \mathrm{min}$./cc. (average $0.036 \pm 0.005$ ); thus the difference between obstruction and vasoconstriction was $0.056 \mathrm{ml}$./ min./cc. 
DIGITAL BLOOD FLOW IN RHEUMATOID ARTHRITIS

TABLE III

DIGITAL BLOOD FLOW IN EIGHT NORMAL CONTROLS AT $20^{\circ} \mathrm{C}$

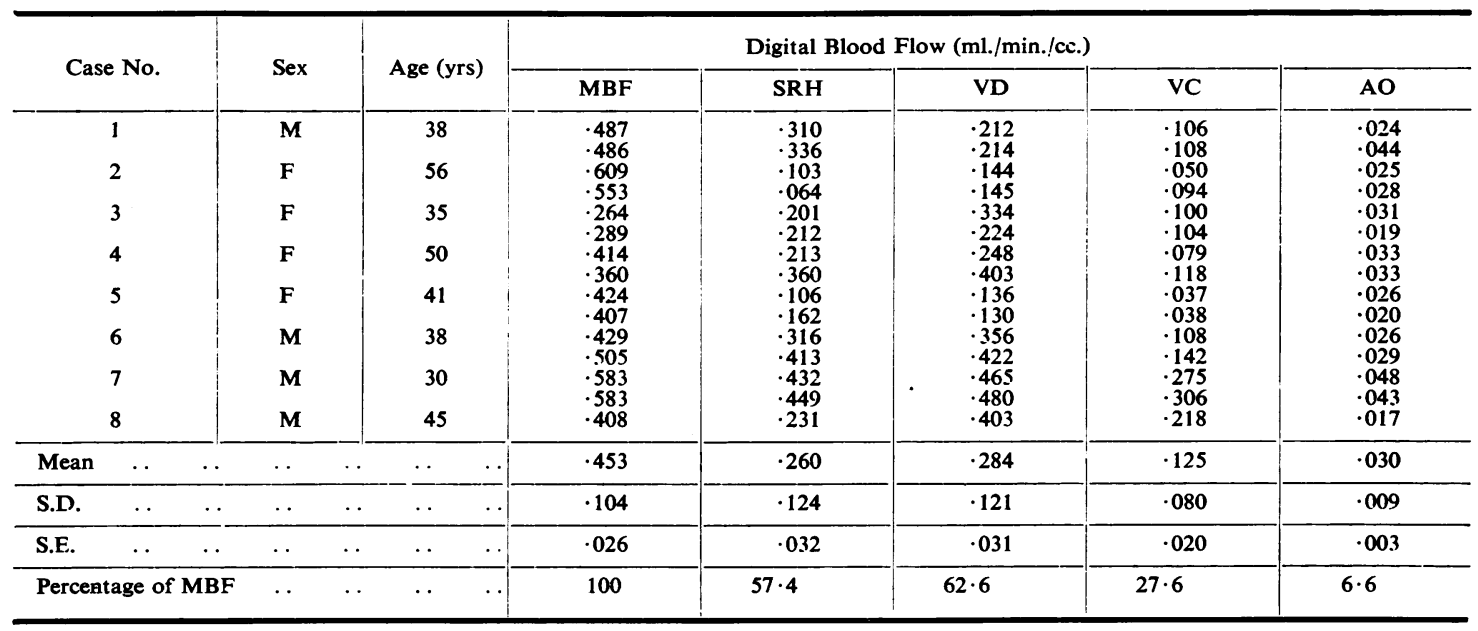

TABLE IV

MEAN DIGITAL BLOOD FLOW IN TWELVE RHEUMATOID ARTHRITICS AND EIGHT NORMAL CONTROLS AT 20 ${ }^{\circ}$ C.

\begin{tabular}{|c|c|c|c|c|c|c|c|c|c|c|c|}
\hline \multirow{2}{*}{ Series } & & \multirow{2}{*}{$\begin{array}{c}\text { No. } \\
\text { of } \\
\text { Cases }\end{array}$} & \multicolumn{2}{|c|}{ Sex } & \multicolumn{7}{|c|}{ DBF (ml./min./cc.) and Equivalent Percentages of MBF } \\
\hline & & & $\mathbf{M}$ & $\mathbf{F}$ & MBF & SRH & VD & VC & AO & VD-VV & VC-AO \\
\hline \multirow[t]{2}{*}{ Rheumatoid Arthritis } & \multirow[t]{2}{*}{$\cdots$} & \multirow[t]{2}{*}{12} & \multirow[t]{2}{*}{4} & \multirow[t]{2}{*}{8} & $\begin{array}{l}.403 \pm \\
.023\end{array}$ & $\begin{array}{l}\cdot 107 \pm \\
.014\end{array}$ & $\begin{array}{l}.117 \pm \\
.023\end{array}$ & $\begin{array}{l}.045 \pm \\
.003\end{array}$ & $\begin{array}{l}.022 \pm \\
.002\end{array}$ & .072 & .023 \\
\hline & & & & & $(100 \%)$ & $(26 \cdot 5 \%)$ & $(29 \cdot 0 \%)$ & $(11 \cdot 1 \%)$ & $(5 \cdot 4 \%)$ & $(17 \cdot 9 \%)$ & $(5 \cdot 7 \%)$ \\
\hline \multirow[t]{2}{*}{ Normal Controls } & & \multirow[t]{2}{*}{8} & \multirow[t]{2}{*}{4} & \multirow[t]{2}{*}{4} & $\begin{array}{r}.453 \\
\pm .026\end{array}$ & $\begin{array}{r}.260 \\
+.032\end{array}$ & $\begin{array}{r}.284 \\
+.031\end{array}$ & $\begin{array}{r}.125 \\
\pm .020\end{array}$ & $\begin{array}{r}.030 \\
\pm .003\end{array}$ & .159 & .092 \\
\hline & & & & & $(100 \%)$ & $(57 \cdot 4 \%)$ & $(64 \cdot 2 \%)$ & $(27 \cdot 6 \%)$ & $(6.6 \%)$ & $(26 \cdot 6 \%)$ & $(21 \cdot 0 \%)$ \\
\hline $\mathbf{P} \quad \ldots$ & $\cdots$ & $\cdots$ & & $\cdots$ & $(\cdot 1-\cdot 2)$ & $(\cdot 001-\cdot 01)$ & $(.001)$ & $(.001)$ & $(\cdot 05-.02)$ & & \\
\hline
\end{tabular}

TABLE V

DIGITAL BLOOD FLOW IN SIX CASES OF RHEUMATOID ARTHRITIS AT $28^{\circ} \mathrm{C}$., BY SEX

\begin{tabular}{|c|c|c|c|c|c|c|c|c|}
\hline \multirow{2}{*}{ Sex } & \multirow{2}{*}{ Case No. } & \multirow{2}{*}{\multicolumn{2}{|c|}{ Age (yrs) }} & \multicolumn{5}{|c|}{$\mathrm{DBF}$ (ml./min./cc.) } \\
\hline & & & & MBF & SRH & VD & VC & AO \\
\hline \multirow[t]{2}{*}{ Male } & $\begin{array}{l}13 \\
14 \\
15\end{array}$ & & $\begin{array}{l}40 \\
39 \\
54\end{array}$ & $\begin{array}{l}\cdot 297 \\
\cdot 295 \\
\cdot 566 \\
\cdot 569 \\
\cdot 304 \\
\cdot 252\end{array}$ & $\begin{array}{l}\cdot 106 \\
\cdot 129 \\
\cdot 110 \\
\cdot 107 \\
\cdot 262 \\
\cdot 261\end{array}$ & $\begin{array}{l}.067 \\
.072 \\
.091 \\
.094 \\
.276 \\
.239\end{array}$ & $\begin{array}{l}.052 \\
.060 \\
.067 \\
.067 \\
.212 \\
\cdot 172\end{array}$ & $\begin{array}{l}.035 \\
.060 \\
.007 \\
.038 \\
.034 \\
.016\end{array}$ \\
\hline & $\begin{array}{l}\text { Mean } \\
\text { S.D. } \\
\text { S.E. }\end{array}$ & $\begin{array}{l}\cdots \\
\cdots \\
\end{array}$ & $\begin{array}{l}\cdots \\
\cdots \\
\cdots\end{array}$ & $\begin{array}{l}\cdot 380 \\
\cdot 146 \\
\cdot 059\end{array}$ & $\begin{array}{l}\cdot 162 \\
.077 \\
.031\end{array}$ & $\begin{array}{l}.137 \\
.092 \\
.037\end{array}$ & $\begin{array}{l}.105 \\
.068 \\
.028\end{array}$ & $\begin{array}{r}.033 \\
.018 \\
.007\end{array}$ \\
\hline \multirow[t]{2}{*}{ Female } & $\begin{array}{l}16 \\
17 \\
18\end{array}$ & & $\begin{array}{l}43 \\
47 \\
62\end{array}$ & $\begin{array}{l}.353 \\
.358 \\
.331 \\
.386 \\
.623 \\
.600\end{array}$ & $\begin{array}{l}\cdot 129 \\
\cdot 139 \\
\cdot 152 \\
\cdot 163 \\
\cdot 153 \\
\cdot 197\end{array}$ & $\begin{array}{l}\cdot 171 \\
\cdot 227 \\
\cdot 098 \\
\cdot 164 \\
\cdot 170 \\
\cdot 160\end{array}$ & $\begin{array}{l}.090 \\
.069 \\
.063 \\
.123 \\
.054 \\
.084\end{array}$ & $\begin{array}{l}.026 \\
.031 \\
.040 \\
.082 \\
.033 \\
.025\end{array}$ \\
\hline & $\begin{array}{l}\text { Mean } \\
\text { S.D. } \\
\text { S.E. }\end{array}$ & $\begin{array}{l}\cdots \\
\cdots \\
\cdots\end{array}$ & $\begin{array}{l}\cdots \\
\cdots \\
\cdots\end{array}$ & $\begin{array}{l}.442 \\
.132 \\
.054\end{array}$ & $\begin{array}{l}.155 \\
.023 \\
.009\end{array}$ & $\begin{array}{l}\cdot 165 \\
\cdot 091 \\
\cdot 037\end{array}$ & $\begin{array}{l}.080 \\
.024 \\
.010\end{array}$ & $\begin{array}{r}.039 \\
.021 \\
.008\end{array}$ \\
\hline Total & $\begin{array}{l}\text { Mean } \\
\text { S.D. } \\
\text { S.E. }\end{array}$ & $\begin{array}{l}\cdots \\
\cdots \\
\cdots\end{array}$ & $\begin{array}{l}\cdots \\
\ldots \\
.\end{array}$ & $\begin{array}{l}.411 \\
.136 \\
.039\end{array}$ & $\begin{array}{l}\cdot 158 \\
.054 \\
.015\end{array}$ & $\begin{array}{l}\cdot 151 \\
.069 \\
.020\end{array}$ & $\begin{array}{l}.092 \\
.048 \\
.014\end{array}$ & $\begin{array}{l}.036 \\
.019 \\
.005\end{array}$ \\
\hline
\end{tabular}


DIGITAL BLOOD FLOW IN FOUR NORMAL CONTROLS AT $28^{\circ} \mathrm{C}$.

\begin{tabular}{|c|c|c|c|c|c|c|c|c|c|c|}
\hline \multirow{2}{*}{\multicolumn{2}{|c|}{ Case No. }} & & \multirow{2}{*}{ Sex } & \multirow{2}{*}{\multicolumn{2}{|c|}{ Age (yrs) }} & \multicolumn{5}{|c|}{ DBF (ml./min./cc.) } \\
\hline & & & & & & MBF & SRH & VD & VC & $\overline{\mathrm{AO}}$ \\
\hline \multicolumn{2}{|c|}{$\begin{array}{l}1 \\
2 \\
3 \\
4\end{array}$} & & $\begin{array}{l}\mathbf{M} \\
\mathbf{M} \\
\mathbf{F} \\
\mathbf{F}\end{array}$ & \multicolumn{2}{|r|}{$\begin{array}{l}30 \\
34 \\
55 \\
41\end{array}$} & $\begin{array}{l}.408 \\
.505 \\
.384 \\
.514 \\
.462 \\
.398 \\
.501 \\
.439\end{array}$ & $\begin{array}{l}\cdot 252 \\
\cdot 321 \\
\cdot 258 \\
\cdot 327 \\
\cdot 250 \\
\cdot 281 \\
\cdot 320 \\
\cdot 300\end{array}$ & $\begin{array}{l}\cdot 303 \\
\cdot 391 \\
\cdot 249 \\
\cdot 281 \\
\cdot 298 \\
\cdot 202 \\
\cdot 424 \\
\cdot 330\end{array}$ & $\begin{array}{l}.212 \\
.256 \\
\cdot 135 \\
\cdot 149 \\
\cdot 200 \\
\cdot 198 \\
\cdot 212 \\
\cdot 148\end{array}$ & $\begin{array}{l}.029 \\
.034 \\
.039 \\
.036 \\
.035 \\
.020 \\
.030 \\
.032\end{array}$ \\
\hline $\begin{array}{l}\text { Mean } \\
\text { S.D. } \\
\text { S.E. }\end{array}$ & $\begin{array}{l}\cdots \\
\cdots \\
\cdots\end{array}$ & $\begin{array}{l}\cdots \\
\cdots\end{array}$ & $\begin{array}{l}\cdots \\
\cdots\end{array}$ & $\begin{array}{l}\cdots \\
\cdots\end{array}$ & $\begin{array}{l}\cdots \\
\cdots \\
\cdots\end{array}$ & $\begin{array}{l}.451 \\
.051 \\
.018\end{array}$ & $\begin{array}{l}.288 \\
.032 \\
.011\end{array}$ & $\begin{array}{l}.315 \\
.068 \\
.024\end{array}$ & $\begin{array}{l}.188 \\
.041 \\
.014\end{array}$ & $\begin{array}{l}.032 \\
.005 \\
.002\end{array}$ \\
\hline \multicolumn{3}{|c|}{ Percentage of MBF } & . & $\ldots$ & $\cdots$ & 100 & $63 \cdot 8$ & $69 \cdot 8$ & $41 \cdot 6$ & $7 \cdot 1$ \\
\hline
\end{tabular}

TABLE VII

MEAN DIGITAL BLOOD FLOW IN SIX CASES OF RHEUMATOID ARTHRITIS AND FOUR NORMAL CONTROLS AT $28^{\circ} \mathrm{C}$.

\begin{tabular}{|c|c|c|c|c|c|c|c|c|c|c|c|c|}
\hline \multirow{2}{*}{\multicolumn{3}{|c|}{ Series }} & \multirow{2}{*}{$\begin{array}{c}\text { No. } \\
\text { of } \\
\text { Cases }\end{array}$} & \multicolumn{2}{|c|}{ Sex } & \multicolumn{7}{|c|}{ DBF (ml./min./cc.) and Equivalent Percentages of MBF } \\
\hline & & & & $\mathbf{M}$ & $\mathbf{F}$ & MBF & SRH & VD & VC & AO & VD-VC & VC-AO \\
\hline \multirow{2}{*}{\multicolumn{2}{|c|}{ Rheumatoid Arthritis }} & & \multirow{2}{*}{6} & \multirow{2}{*}{3} & \multirow{2}{*}{3} & .411 & $\cdot 158$ & $\cdot 151$ & .092 & .036 & .059 & .056 \\
\hline & & & & & & $(100 \%)$ & $(38 \cdot 4 \%)$ & $(36 \cdot 7 \%)$ & $(22 \cdot 4 \%)$ & $(8 \cdot 7 \%)$ & $(14 \cdot 3 \%)$ & $(13 \cdot 7 \%)$ \\
\hline \multirow{2}{*}{ Normal Controls } & \multirow{2}{*}{. } & & \multirow{2}{*}{4} & \multirow{2}{*}{2} & \multirow{2}{*}{2} & .451 & .288 & $\cdot 315$ & $\cdot 188$ & .032 & .127 & $\cdot 156$ \\
\hline & & $\cdots$ & & & & $(100 \%)$ & $(63 \cdot 8 \%)$ & $(69 \cdot 8 \%)$ & $(41 \cdot 6 \%)$ & $(7 \cdot 1 \%)$ & $(28 \cdot 2 \%)$ & $(34.5 \%)$ \\
\hline $\mathbf{P} \quad \ldots$ & $\ldots$ & $\ldots$ & .. & & & .4 & .001 & .001 & .001 & 0.8 & & \\
\hline
\end{tabular}

The corresponding figures for four normal controls are given in Table VI.

Table VII summarizes the DBF values for rheumatoid cases and normal controls at temperature $28^{\circ} \mathrm{C}$., as well as the percentages calculated as described above.

\section{Correlations of Results with Various Factors}

An attempt was made to correlate the changes in DBF in rheumatoid cases at the temperature of $20^{\circ} \mathrm{C}$. in different phases of the test with the following factors which were thought to be relevant:

(1) Sex.

(2) Duration or chronicity of the disease (arthritis).

(3) Anatomical stage of the lesion.

(4) Titre of Waaler-Rose test (Greenbury, 1957).

For the MBF, values of DBF in actual ml./min./ c.c. were used, whereas, for the other phases percentage values were thought to represent a better comparison of the types of behaviour (Arab, 1964).

(1) Sex.-Table VIII and Fig. 2 (opposite) show the difference between the two sexes in cold and warm temperatures separately. Thus, at $20^{\circ} \mathrm{C}$., the average MBF for females was about the same as that for males (only insignificantly higher), yet the percentage values at all the other phases were significantly lower in females, especially at SRH and VD. The vasodilatation-vasoconstriction wave was also less in the females. At $28^{\circ} \mathrm{C}$. the average MBF for females was a little above that for males, but the percentage values at the other phases showed no persistently significant difference.

(2) Duration of Rheumatoid Arthritis.-No correlation could be established between the duration of the disease and either the MBF or other flow values at other phases.

(3) Stage of Rheumatoid Arthritis.-Table IX and Fig. 3 (opposite) show the average DBF values for the twelve rheumatoid cases tested at $20^{\circ} \mathrm{C}$., divided into three groups according to the anatomical stage of the arthritis.

Thus the more advanced the lesion the less was the MBF after indirect heating. The same relationship was seen when the percentage values for vasodilatation at rest or the vasoconstriction-vasodilatation wave were compared at the three stages.

(4) Differential Agglutination Titre.-For eleven cases a titre was recorded in the Waaler-Rose test 
TABLE VIII

MEAN DIGITAL BLOOD FLOW IN RHEUMATOID ARTHRITICS AT DIFFERENT TEMPERATURES, BY SEX

\begin{tabular}{|c|c|c|c|c|c|c|c|c|}
\hline \multirow{2}{*}{ Temp. } & \multirow{2}{*}{ Sex } & \multirow{2}{*}{$\begin{array}{c}\text { No. } \\
\text { of } \\
\text { Cases }\end{array}$} & \multirow{2}{*}{$\underset{\text { (ml./min./cc.) }}{\mathrm{MBF}}$} & \multicolumn{5}{|c|}{ Mean DBF as Percentage of MBF } \\
\hline & & & & SRH & VD & VC & AO & VD-VC \\
\hline & Male & 4 & $\begin{array}{l}.386(100 \%) \\
\pm .054\end{array}$ & $\begin{array}{l}38.6 \% \\
\pm 6.9 \\
\end{array}$ & $\begin{array}{l}43 \cdot 0 \% \\
\pm 9 \cdot 7\end{array}$ & $\begin{array}{l}15 \cdot 4 \% \\
\pm 2.5 \\
\end{array}$ & $\begin{aligned} & 9.0 \% \\
& \pm 1.5 \\
&\end{aligned}$ & $27 \cdot 6 \%$ \\
\hline \multirow[t]{3}{*}{20} & Female & 8 & $\begin{array}{l}.412(100 \%) \\
\pm .023\end{array}$ & $\begin{array}{l}21 \cdot 9 \% \\
\pm 2 \cdot 7\end{array}$ & $\begin{array}{l}21 \cdot 5 \% \\
\pm 3.9\end{array}$ & $\begin{aligned} & 9 \cdot 7 \% \\
\pm & 1 \cdot 5\end{aligned}$ & $\begin{aligned} & 3.0 \% \\
\pm & 0.7\end{aligned}$ & $11 \cdot 8 \%$ \\
\hline & $\mathbf{P}$ & $\bar{\cdots}$ & .05 & .05 & .05 & .05 & .01 & \\
\hline & Male & 3 & $\begin{array}{r}\cdot 380(100 \%) \\
\pm \cdot 059\end{array}$ & $42 \cdot 6 \%$ & $36.0 \%$ & $27 \cdot 6 \%$ & $8 \cdot 7 \%$ & $8 \cdot 4 \%$ \\
\hline \multirow[t]{2}{*}{28} & Female & 3 & $\begin{array}{l}.442(100 \%) \\
.054\end{array}$ & $35 \cdot 1 \%$ & $37 \cdot 3 \%$ & $18 \cdot 1 \%$ & $8 \cdot 8 \%$ & $9 \cdot 2 \%$ \\
\hline & $\mathbf{P}$ & . & $\cdot 4$ & .05 & .05 & .05 & .05 & \\
\hline
\end{tabular}
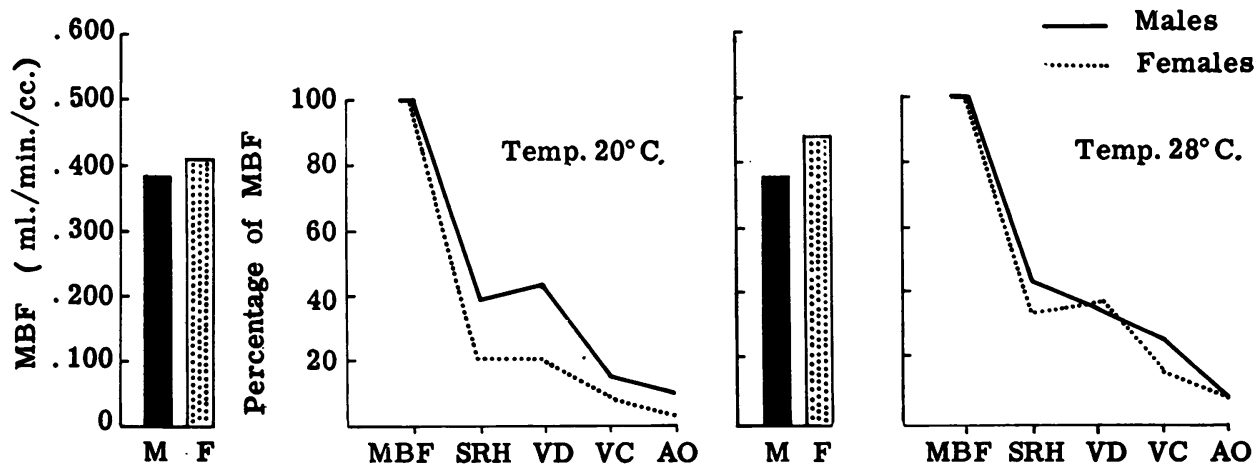

Fig. 2.-Sex difference in digital blood flow in rheumatoid arthritis.

TABLE IX

MEAN DIGITAL BLOOD FLOW IN RHEUMATOID ARTHRITICS AT $20^{\circ}$ C., BY STAGE OF ARTHRITIS

\begin{tabular}{|c|c|c|c|c|c|c|c|c|c|}
\hline \multirow{2}{*}{$\begin{array}{c}\text { Stage } \\
\text { of } \\
\text { Arthritis }\end{array}$} & \multirow{2}{*}{$\begin{array}{l}\text { No. } \\
\text { of } \\
\text { Cases }\end{array}$} & \multicolumn{2}{|c|}{ Sex } & \multirow{2}{*}{$\underset{(\mathrm{ml} . / \mathrm{min} . / \mathrm{cc} .)}{\mathrm{MBF}}$} & \multicolumn{5}{|c|}{ Mean DBF as Percentage of MBF } \\
\hline & & $\mathbf{M}$ & $\mathbf{F}$ & & SRH & VD & VC & $\mathbf{A O}$ & VD-VC \\
\hline $\mathbf{J}$ & 1 & 0 & 1 & $0.530(100 \%)$ & $50 \cdot 5$ & $54 \cdot 1$ & $22 \cdot 4$ & $1 \cdot 6$ & $31 \cdot 7$ \\
\hline II & 5 & 1 & 4 & $0.438(100 \%)$ & $21 \cdot 1$ & $31 \cdot 0$ & $10 \cdot 2$ & $4 \cdot 7$ & $20 \cdot 8$ \\
\hline III & 6 & 3 & 3 & $0.352(100 \%)$ & $29 \cdot 7$ & $21 \cdot 7$ & $10 \cdot 9$ & $6 \cdot 6$ & $10 \cdot 8$ \\
\hline
\end{tabular}
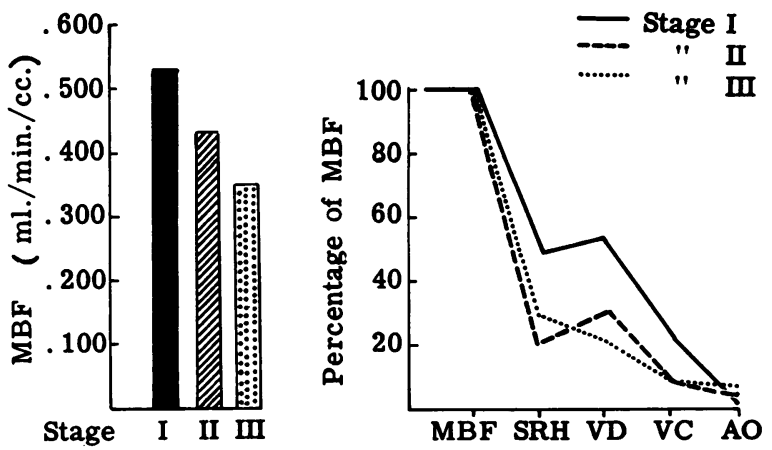

Fig. 3.-Relationship between digital blood flow and stage of rheumatoid arthritis. 
as modified by Greenbury (1957). Table X shows the relationship between these titres and the digital blood flows. The standard reactive hyperaemia expressed as a percentage of the maximal blood flow was greater in the group with the higher titres. The difference between the vasoconstriction and vasodilatation waves at room temperature was also greater in this group, when expressed in similar terms, than the group in the lower titre range.

These findings show that the blood vessels are less responsive in the lower titre group than the higher titre group, which includes the chronologically earlier cases.

\section{Discussion}

The question whether the above figures representing digital blood flows as calculated in this method give true values for digital blood flows, has been discussed previously (Arab, 1964). However, this need be of no great concern here, as the same method for the calculation was used for the cases under study, and the controls. Again, the use of percentage values in comparing the patterns of behaviour of particular groups at different stages eliminates errors due to calculations if these figures were representing only a fraction of the true blood flow.

The comparison between rheumatoid cases and normal controls, as regards their maximal digital blood flow after full sympathetic release by indirect body heating, shows that they fall within the same range at this particular state. It should be noted in this connection, that this only holds when comparison is made with controls of similar age, i.e. above 30 years: it has been shown previously that the digital blood flows tend to be lower with advancing age in healthy individuals (Arab, 1964).

The success in obtaining a good vasomotor response to indirect heating in nearly all the cases studied here, was probably due to the efficiency of the method used which involved immersing about 15-20 per cent. of the body surface in hot water. Fail. - to achieve release of vasomotor tone may explain previous reports of failure to get a complete response in some rheumatoid cases (Woodmansey, 1951), although we cannot exclude the presence of an organic vascular occlusive state in those cases which would not be released by indirect heating.

In contrast to the above results, during the resting state before body heating, rheumatoid cases behaved quite differently from normals. Thus, at the temperature of $20^{\circ} \mathrm{C}$. they showed a greater tendency to an increased vascular tone, as evidenced by the significantly lower values of resting vasodilatation flows, the narrower vasodilatationconstriction waves, and the level of vasoconstriction flows being nearer to the obstructed state (Fig. 4, opposite).

So the general behaviour of the digital circulation in rheumatoid arthritis would fall midway between their appropriate controls and that observed in classical cases of Raynaud's disease (Fig. 5, opposite).

The latter have a very marked vascular tone at a temperature of $20^{\circ} \mathrm{C}$., which was manifested by a characteristic pattern of very poor resting vasodilatation flows, almost absent vasodilatationconstriction waves and resting vaso-constriction flows which were very close to those obtained during obstruction of the circulation (Arab, 1964).

At the warmer temperature of $28^{\circ} \mathrm{C}$., the deviation fron $\iota$ the normal pattern of behaviour described above for rheumatoid cases is still manifest, which shows that the increased vascular tone in rheumatoid cases is present even at a comfortably warm temperature.

Sex has been shown to play a significant role, since females had a pattern nearer to the Raynaud's cases, while males were more normal. This could be demonstrated clearly by comparing the pattern of the digital circulation at different phases before indirect heating, expressed as percentages (Fig. 2). After indirect body heating the maximal digital flows were about the same; in fact, a little higher in females. This finding goes well with the known fact that peripheral circulatory disturbances in the hands and Raynaud's phenomena are more frequent

TABLE X

DIFFERENTIAL AGGLUTINATION TITRE (D.A.T.) AND DIGITAL BLOOD FLOW

\begin{tabular}{|c|c|c|c|c|c|c|c|c|c|}
\hline \multirow{2}{*}{ D.A.T. } & \multirow{2}{*}{$\begin{array}{c}\text { No. } \\
\text { of } \\
\text { Cases }\end{array}$} & \multicolumn{2}{|c|}{ Sex } & \multirow{2}{*}{ MBF } & \multirow{2}{*}{ SRH } & \multirow{2}{*}{ VD } & \multirow{2}{*}{$\mathrm{VC}$} & \multirow{2}{*}{ AO } & \multirow{2}{*}{ VD-VC } \\
\hline & & $\mathbf{F}$ & $\mathbf{M}$ & & & & & & \\
\hline \multirow{2}{*}{ Under $1: 32$} & \multirow{2}{*}{6} & \multirow{2}{*}{4} & \multirow{2}{*}{2} & $0 \cdot 397$ & 0.081 & 0.067 & 0.030 & 0.031 & 0.037 \\
\hline & & & & $100 \%$ & $24 \%$ & $0.7 \%$ & $7 \cdot 6 \%$ & $7 \cdot 8 \%$ & $9 \cdot 3 \%$ \\
\hline \multirow{2}{*}{$1: 32$ and Over } & \multirow{2}{*}{5} & \multirow{2}{*}{3} & \multirow{2}{*}{2} & 0.427 & $0 \cdot 145$ & $0 \cdot 185$ & 0.065 & 0.023 & $0 \cdot 120$ \\
\hline & & & & $100 \%$ & $34 \%$ & $43 \%$ & $15 \%$ & $5 \cdot 4 \%$ & $28 \%$ \\
\hline
\end{tabular}


DIGITAL BLOOD FLOW IN RHEUMATOID ARTHRITIS

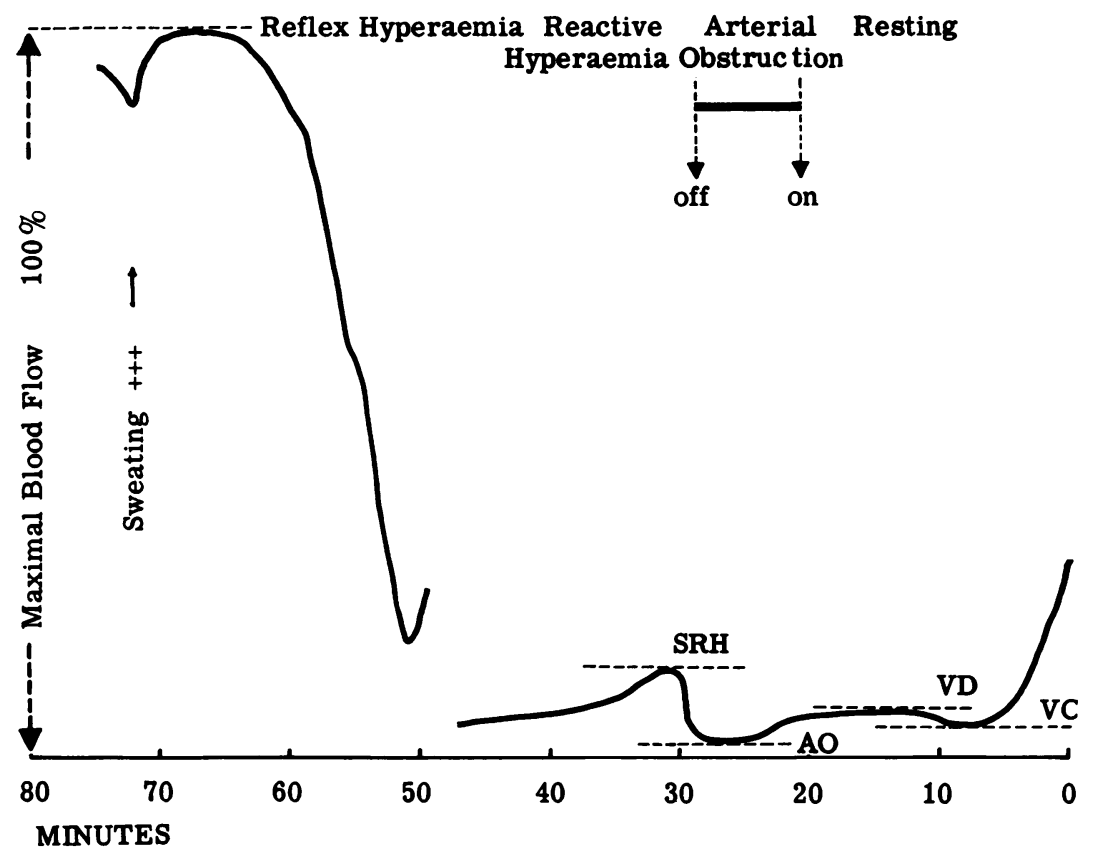

Fig. 4.-Record of digital blood flow in a rheumatoid patient at $20^{\circ} \mathrm{C}$.

Reflex Hyperaemia

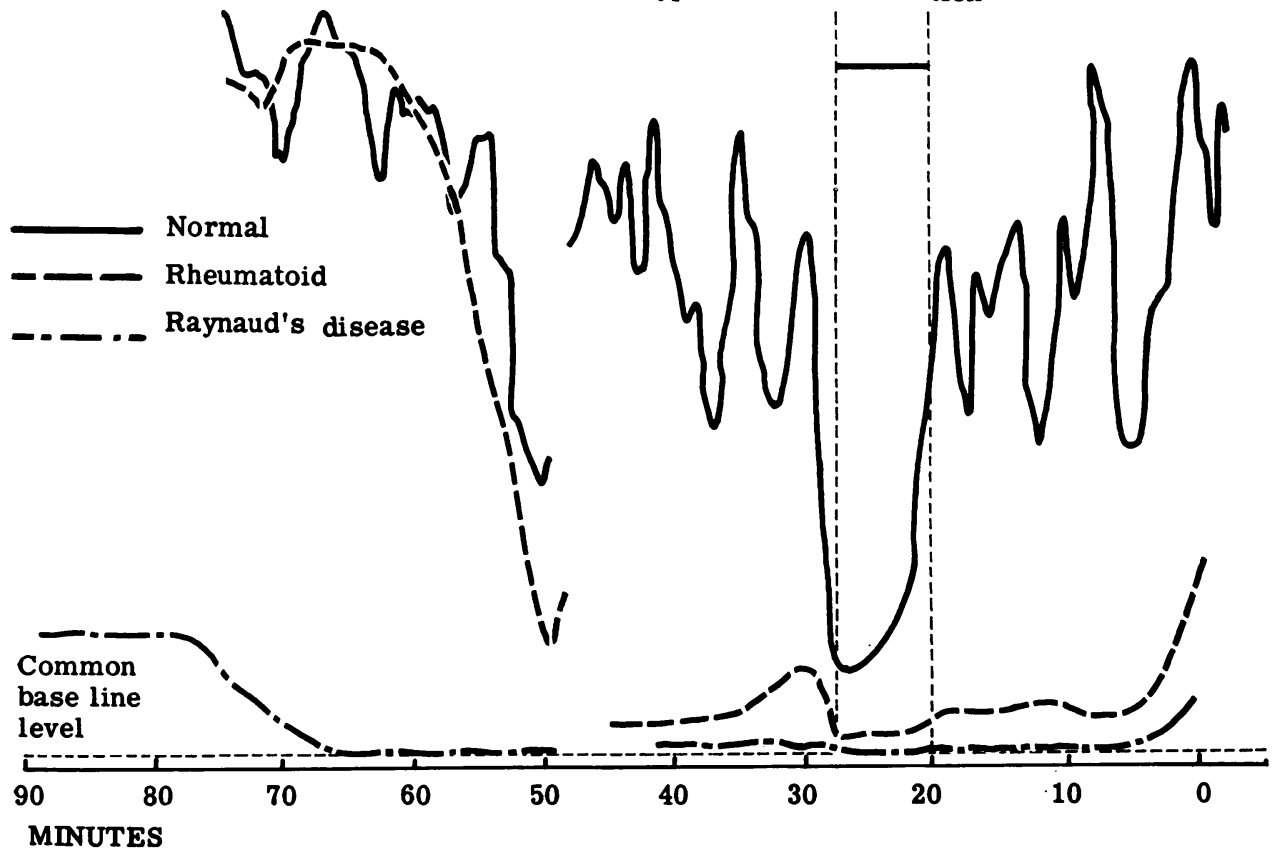

Reactive Arterial Resting Hyperaemia Obstruction

Fig. 5.-Records of digital blood flow in a normal subject and in a patient with Raynaud's disease, showing differences in general pattern from that of the patient recorded in Fig. 4, here superimposed. 
in females, and, may possibly be of significance in explaining the higher incidence of rheumatoid arthritis as a whole in females than in males.

The successful correlation of the extent of joint lesion with the total blood flow after indirect heating shows that there is more tendency towards a vascular occlusive state with more advanced lesions. However, it is also shown that the more advanced the lesion, the greater the tone in the digital circulation, as evidenced by reduced percentage resting vasodilatation, as well as smaller VD-VC waves. The duration of the disease is found to have no influence on the digital blood flow, and this also goes well with the known fact that even the arthritic lesion itself progresses at different rates in different individuals.

\section{Summary}

The digital circulation in cases of rheumatoid arthritis has been studied by a continuous flow calorimetry technique. Under a cold temperature of $20^{\circ} \mathrm{C}$., it was shown to have a pattern midway between that seen in normal subjects and in cases of Raynaud's disease, i.e. there is a high vasoconstrictive tone and a poor vasodilatation-vasoconstriction wave. The abnormality was more manifest in females than in males and with more advanced than with early arthritic lesions.

We wish to acknowledge our thanks to the Empire Rheumatism Council for a-grant to purchase the recording galvanometer used in these studies; to Professor W. J. H. Butterfield for his guidance and encouragement and for making his calorimeters available to us; and to Mr. Brian Cox and Miss D. Bruce for technical assistance.

\section{REFERENCES}

Arab, M. M. H. (1964). A calorimetric study of the digital blood flow in normal subjects and some abnormal vascular and non-vascular conditions. To be published in Guy's Hospital Reports.

Aronoff, A., Johnson, L., and Dworkin, S. (1960). Canad. med. Ass. J., 83, 58.

Beattie, J. W., and Woodmansey, A. (1953). Ann. rheum. Dis., 12, 43.

Butterfield, W. J. H. (1964). Guy's Hosp. Rep. (to be published).

Bywaters, E. G. L. (1957). Ann. rheum. Dis., 16, 84.

Catchpole, B. N., Jepson, R. P., and Kellgren, J. H. (1954). Ibid., 13, 302.

Copeman, W. S. C. (1955). "Textbook of the Rheumatic Diseases", 2nd ed., p. 166. Livingstone, Edinburgh.

Cruickshank, B. (1954). Ann. rheum. Dis., 13, 136.

Empire Rheumatism Council (1950). "Report by Scientific Advisory Committee." Brit. med. J., 1, 799.

Graef, I., Hickey, D. V., and Altmann, V. (1949). Amer. Heart J., 37, 635.
Greenbury, C. L. (1957). Broadsheet No. 18 (new series) of the Association of Clinical Pathologists.

Levin, M. H., Rivo, J. B., Scott, W., Figueroa, W. G., Fred, L., and Barrett, T. F. (1953). Amer. J. Med., 14, 265.

Mendlowitz, M. (1950). Angiology, 1, 247.

Morgan, G. (1959). Ann. rheum. Dis., 18, 322.

Report of the Medical Research Council and the Nuffield Foundation (1955). Brit. med. J., 2, 695.

Schmid, F. R., Cooper, N. S., Ziff, M., and McEwen, C. (1961). Amer. J. Med., 30, 56.

Scott, J. T., Hourihane, D. O., Doyle, F. H., Steiner, R. E., Laws, J. W., Dixon, A. St. J., and Bywaters, E. G. L. (1961). Ann. rheum. Dis., 20, 224.

Sokoloff, L., Wilens, S. L., and Bunim, J. J. (1951). Amer. J. Path., 27, 157.

Steinbrocker, O., Traeger, C. H., and Batterman, R. C. (1949). J. Amer. med. Ass., 140, 659.

Virtama, P. (1959). Acta rheum. scand., 5, 304.

Woodmansey, A. (1951). Ann. rheum. Dis., 10, 65.

\section{DISCUSSION}

DR. J. T. ScotT (Hammersmith): This application of physiological techniques to rheumatoid arthritis is very interesting. I should like to ask two questions:

(1) With regard to the sex difference which has been demonstrated, was a similar incidence found in the control group?

(2) Have you any ideas about the correlation of this type of functional change and the anatomical changes that we and others have demonstrated in digital vessels in rheumatoid arthritis?

DR. Holden: The sex differences were found in rheumatoid arthritis cases only. There is no doubt that vascular changes were more common in arthritic females than in males. The patients tested showed no clinical evidence of a vascular abnormality, so, in that respect, they differed from those tested by others.

With regard to the second question, none of the patients tested were in an advanced stage of the disease, because the difficulty of getting crippled patients into and out of a hip bath ruled out the more advanced stages of the disease and probably explains why only functional changes were found in the digital blood vessels in our patients. It would be interesting to carry out further studies on patients with more advanced disease.

Prof. E. G. L. Bywaters (Taplow): Can Dr. Holden tell us something of the significance of the differences he showed? He quoted only averages and not standard deviations. I find it difficult, for instance, without this to understand the importance of the association between blood flow changes and the Stages I, II, and III of disease. There was only one case in Stage I. Is this a valid statistical difference between Stages I, II and III?

DR. Holden: It is quite true that we only had one case in Stage I, and I do not claim that the differences here are statistically significant. The statistics are 
conclusive regarding the figures shown for sex difference and also for temperature changes.

Prof. J. Michez (Brussels): This study suggests that a comparison should be made between rheumatoid arthritis and degenerative joint disease. I think there is a great difference between the two. It is interesting to see that in the rheumatoid group a peak exists after the vasoconstriction and vasodilatation. In degenerative joint disease we sometimes see the contrary, in that there is too much response to vasoconstriction and vasodilatation.

\section{Etude calorimétrique du courant sanguin digital dans} l'arthrite rhumatismale

\section{RÉSUMÉ}

On étudia la circulation digitale des cas d'arthrite rhumatismale par un procédé calorimétrique à courant continu. A la température de $20^{\circ} \mathrm{C}$. on observa un tableau intermédiaire entre celui pour des sujets normaux et celui pour des cas de maladie de Raynaud, c'est à dire il y eut un fort tone vasoconstricteur et une onde faible de vasodilatation-constriction. Cette anomalie fut plus en évidence chez des femmes que chez des hommes et plus manifeste dans des cas de lésions arthritiques avancées.

Estudio calorimétrico del corriente sanguíneo digital en la artritis reumatoide

\section{SUMARIO}

Se estudió la circulación digital de casos de artritis reumatoide por un procedimiento calorimétrico a corriente contínua. A la temperatura de $20^{\circ} \mathrm{C}$. se ve un cuadro intermedio entre el observado en sujetos normales y aquel en la enfermedad de Raynaud, es decir un fuerte tono vasoconstrictor y una debil onda de vasodilatación-constricción. Esta anomalía fué más manifesta en mujeres que en hombres y más pronunciada en casos de lesiones artríticas avanzadas. 